\title{
Lensless Digital In-line Holographic Microscopy for Space Biotechnology Applications
}

\author{
Kerem Delikoyun \\ Department of Bioengineering \\ Izmir Institute of Technology \\ Izmir, TURKEY \\ keremdelikoyun@iyte.edu.tr \\ Mustafa Ozuysal \\ Department of Computer Engineering \\ Izmir Institute of Technology \\ Izmir, TURKEY \\ mustafaozuysal@iyte.edu.tr
}

\author{
Ersin Cine \\ Department of Computer Engineering \\ Izmir Institute of Technology \\ Izmir, TURKEY \\ ersincine@iyte.edu.tr \\ Engin Ozcivici \\ Department of Bioengineering \\ Izmir Institute of Technology \\ Izmir, TURKEY \\ enginozcivici@iyte.edu.tr
}

\author{
Muge Anil-Inevi \\ Department of Bioengineering \\ Izmir Institute of Technology \\ Izmir, TURKEY \\ mugeanil@iyte.edu.tr \\ H. Cumhur Tekin \\ Department of Bioengineering \\ Izmir Institute of Technology \\ Izmir, TURKEY \\ cumhurtekin@iyte.edu.tr
}

\begin{abstract}
Biomechanical changes at cellular level can dramatically affect living organisms in both aviation and space applications. Weightlessness induces morphological alteration of cells, which leads to tissue loss. Therefore, scientists have been studying the effect of weightlessness using cell culture based biological experiments using conventional microscopes. However, strict requirements regarding cost, weight and functionality limit the use of conventional microscopes in space environment. Lensless digital in-line holographic microscopy enables to use low-weight, low-cost and robust elements, such as a light emitting diode (LED), an aperture and an imaging sensor, instead of bulky, expensive and fragile optical elements, such as lenses, mirrors and filters. This technology offers a high field of view compared to conventional microscopes without affecting the resolution and it is also suitable for remote sensing applications with automated imaging capabilities. Here, we present a portable digital in-line holographic microscopy platform that allows to visualize cells and to analyze their viability in a microfluidic chip. The platform offers microscopic imaging with $1.55 \mu \mathrm{m}$ spatial resolution, $21.7 \mathrm{~mm}^{2}$ field of view and image coloring capability. This platform could potentially play an important role in space biotechnology applications by enabling low-cost, high-resolution and portable monitoring of cells.
\end{abstract}

Keywords - digital in-line holographic microscopy, lensless imaging, cell culture, viability

\section{INTRODUCTION}

Biological adaptations to space environment are important determinants of long-term human presence in space missions. Biomechanical changes induced by weightlessness are known to affect many organs [1, 2]. Furthermore, without the protective environment sustained with the atmosphere, organisms are exposed to much higher background radiation that may damage DNA [3]. It is therefore important to establish biological research infrastructure in space stations.

Research related to biological adaptations to space environment can be modeled on the earth using in vitro [4] and in vivo $[5,6]$ approaches. However, the gold standard is performing the research in the space. For a long time, scientist have been done variety of biological experiments in International Space Station (ISS) by using cell cultures that are monitored by video microscopy to observe morphological changes. Microscopy is one of the greatest tools in biological sciences among many other disciplines that offers the capability of examining the micro/nano size objects. However, microscopes are expensive tools, require trained personnel to operate and more importantly they are bulky and heavy. Launching costs for a kilogram of payload to orbit exceeds $\$ 5000$ in 2016 [7], signifying the importance of the need for ultra-light weight imaging equipment.

In the last decade, digital in-line holographic microscopy has been an emerging field in optical imaging. Further advances have been achieved in this field by replacing expensive and bulky optics (e.g. lenses, mirrors, filters and sophisticated lighting equipment) with an aperture and incoherent light source (light emitting diode - LED) [8]. This system provides coherency by filtering incoherent light of LED through the aperture. In lensless digital in-line holographic microscopy, a sample is directly placed on the imaging sensor (i.e., complementary metal oxide semiconductor - CMOS). Therefore, entire area of imaging sensor can be utilized with a field of view (FOV) of $\sim 20 \mathrm{~mm}^{2}$ and spatial resolution of smaller than $2 \mu \mathrm{m}$ [9]. This FOV of lensless digital in-line holographic microscopy is more than 50 times larger than conventional microscope systems with similar magnification characteristics $(40 \times$ objectives). Since bulky and sensitive optical components are not required, lensless microscopy offers compact, portable and low-cost imaging solutions that can be potentially adapted for almost all kind of environments, including space stations.

In this study, we present a portable lensless digital in-line holographic microscopy system for monitoring cells and conducting viability experiments that could be used for space based in vitro experiments. Among other microscopic techniques, lensless digital in-line holographic microscopy offers quantitative, high resolution, easy-to-use and remote imaging capabilities. The system could also be adapted to screen health status of astronauts from a finger-prick blood sample.

\section{EXPERIMENTAL METHODS}

\section{A. Biological Sample Preparation}

D1 ORL UVA bone marrow stem cells [10] were used in the study. First, D1 cells at the concentration of $2 \times 10^{6}$ cells $/ \mathrm{mL}$ were diluted by using phosphate buffer saline (PBS) and consecutively stained by Trypan blue for testing cell viability. After loading cell solution $\left(\begin{array}{ccc}\sim & \mu \mathrm{L}\end{array}\right)$ into 
microfluidic chip, cells were rested to reach a steady state position at the bottom surface the chip that leads to a more convenient focus of cell layer at the same depth of field when imaged in the microscope.

\section{B. Microfluidic Chip}

Microfluidic chip is composed of three layers, which are glass slide at the bottom, double side adhesive (DSA) tape at the middle and Poly(methyl methacrylate) (PMMA) at the top (Fig. 1a). Glass slide was engraved to have grid-structure by laser cutter (Epilog Zing 24 Laser Cutter). DSA and PMMA were processed to define microfluidic channels using the laser cutter. Inlets and outlets of microfluidic channels were also opened on the PMMA layer. After all layers were processed, DSA was aligned and placed on PMMA and eventually, glass slide was adhered on DSA layer.

\section{Lensless Digital In-Line Holographic Microscopy}

Lensless digital in-line holographic microscopy platform is composed of CMOS imaging sensor and a light source to visualize cells in microfluidic chips (Fig. 1b). AR1820HS (ON Semiconductors) was used as CMOS imaging sensor that has $1.1 \mu \mathrm{m}$ in pixel size with $4912(\mathrm{H})$ pixel $\times 3648(\mathrm{~W})$ pixel of sensor active area $\left(21.7 \mathrm{~mm}^{2}\right)$. Light source was composed of an LED whose central wavelength is $650 \mathrm{~nm}$ and a collimator coupled with optical fiber having a core diameter of $400 \mu \mathrm{m}$ (Ocean Optics). Microfluidic chip was placed on the imaging sensor. Distance between fiber tip to microfluidic chip was about $15 \mathrm{~cm}$. After capturing images on the CMOS sensor, recorded digital holograms $\left(\Psi_{P o}(x, y)\right)$ were reconstructed solving following equation:

$$
\Psi_{P}(x, y ; z)=\mathcal{F}^{-1}\left\{\mathcal{F}\left\{\Psi_{P o}(x, y)\right\} H\left(k_{x}, k_{x} ; z\right)\right\}
$$

where $H\left(k_{x}, k_{x} ; z\right)$ is the spatial transfer function:

$$
H[p, q]=\exp \left[-j k_{o} z \sqrt{1-\frac{\left(p \Delta_{k x}\right)^{2}}{k_{o}^{2}}-\frac{\left(q \Delta_{k y}\right)^{2}}{k_{o}^{2}}}\right]
$$

$k_{o}=w_{o} / v$ is the wave number, where $w_{o}$ is angular frequency $(\mathrm{rad} / \mathrm{s})$ and $v$ is the speed of the wavefront. $(x, y)$ and $(p, q)$ are indices of the sample at the spatial domain and Fourier domain, respectively. $\Delta_{k x}=2 \pi / M \Delta_{x}$ and $\quad \Delta_{k y}=$ $2 \pi / N \Delta_{y}$ are frequency resolutions (radian per unit of length), where $\Delta_{x}$ and $\Delta_{x}$ are sampling periods, and $\mathrm{M}, \mathrm{N}$ are the number of samples in the direction of $\mathrm{x}$ and $\mathrm{y}$ respectively. $\mathrm{z}$ is the distance between sample and imaging sensor. Since exact $\mathrm{z}$ distance is not known, $\mathrm{z}$ is scanned digitally to find focus plane of the object. Reconstructed images $\left(\Psi_{P o}(x, y ; z)\right)$ are complex-valued so that they compose of real and imaginary parts. Amplitude and phase values of complex-valued images are also calculated.

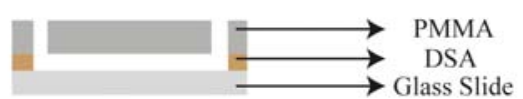

b

Optical Fiber

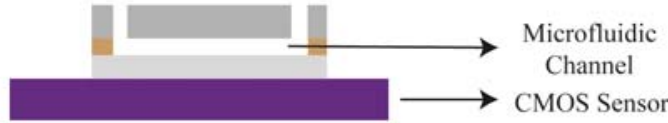

Fig. 1. Illustrations of (a) microfluidic chip and (b) digital in-line holographic microscopy platform.

\section{RESUlts AND DiscUSSION}

We conducted cell viability assay in a microfluidic chip. To do so, Trypan blue staining was used for identifying live/dead cells, which is a common procedure for cell culture process. Stained D1 ORL UVA bone marrow stem cells were imaged under a brightfield inverted microscope (Zeiss Axio Vert A1) with $20 \times$ objective (Fig. 2a) and also our lensless digital in-line holographic microscopy platform (Fig. 2b). Amplitude and phase images (Fig. 2c, d) of reconstructed hologram images were obtained.

Trypan blue dye stains dead cells to blue and live cells remain unstained that can be easily noticed under the brightfield microscope (Fig. 2a). Moreover, live/dead cells were easily distinguishable in reconstructed holograms. For instance, in the amplitude image of reconstructed hologram (Fig. 2c), dead cell was seen as blurry compared to live cells. Also notice that in the phase image (Fig. 2d), intensity of the dead cell was remarkably different than live cells.

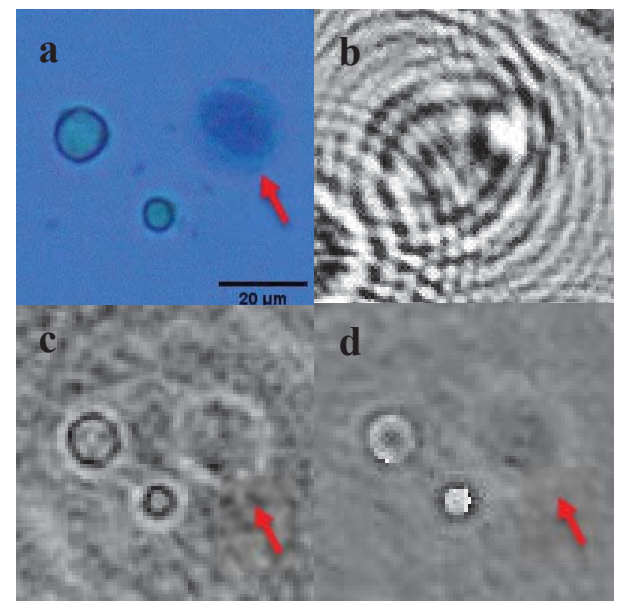

Fig. 2. Inspections of live and dead cells. (a) Brightfield microscope and (b) holographic images of cells. (c) Amplitude and (d) phase images of reconstructed hologram, and (d) phase images of reconstructed hologram. Arrows show dead cells on the images. 
In amplitude and phase images, mean intensity values within each cell were analyzed in Table I. Mean intensity values of dead/live cells were similar in amplitude images. However, live cells showed higher intensity values than the dead cell in the phase image. Therefore, phase intensity values could be used to identify dead/live cells.

TABLE I. Comparison of Mean Intensity Values of Reconstructed Hologram Images

\begin{tabular}{|c|c|c|c|}
\hline \multirow{2}{*}{ Images } & \multicolumn{3}{|c|}{ Mean Intensity Values } \\
\cline { 2 - 4 } & Live cell 1 & Live cell 2 & Dead cell \\
\hline Amplitude & 133.58 & 121.84 & 124.04 \\
\hline Phase & 150.69 & 191.70 & 121.09 \\
\hline
\end{tabular}

We colored the image captured by using lensless digital in-line holographic microscopy platform with amplitude and phase images of reconstructed hologram. Each three channel of the colored image was assigned as amplitude, average of amplitude and phase, and phase of the reconstructed hologram, respectively. Thus, difference between dead and live cells can be more clearly seen (Fig. 3a). Color contrast can be further improved by changing the weights of each channel and using different color spaces such as YUV or HSV.

Dead cells lose their structural stability of their membranes so that Trypan blue dye passes through the cell membrane. On the other hand, membranes of live cells are intact and Trypan blue dye cannot pass through the membrane. Hence, there is a distinct color difference between the interior and exterior of live cells. We observed intact cell membranes in amplitude images as circular low intensity values around the cells (Fig. $3 b)$. The intensity profiles were analyzed on the cells through line segments (Fig. 3b). Minimum intensity values on the profiles showed location of live cells' membranes (Fig. 3c, d), whereas membrane of the dead cell was not clearly distinguishable (Fig. 3e). We averaged the intensity profiles on 180-line segments generated by rotating line segments shown in Fig $3 \mathrm{~b}$ around their centers. Similar intensity profiles are obtained compared (line plots on Fig. 3c-e) to single line segments (dotted plots on Fig. 3c-e). Therefore, intensity profiles on the cells were highly robust to rotations of the line segments.

As shown in Fig. 4, a standard optical resolution chart USAF 1951 was also imaged to determine the resolution of our lensless digital in-line holographic microscopy. Magnified region in Fig. 4a contains group 8 and 9. Vertical and horizontal lines on element 3 of group 8 were distinguishable with the lensless imaging system. Three parallel lines with a half pitch of $1.55 \mu \mathrm{m}$ were observed on the intensity profiles as local intensity values (Fig. 4b, c). Hence, $1.55 \mu \mathrm{m}$ spatial resolution was reached with this imaging platform. To improve further spatial resolution, multi-frame super resolution algorithms [8] could be implemented.
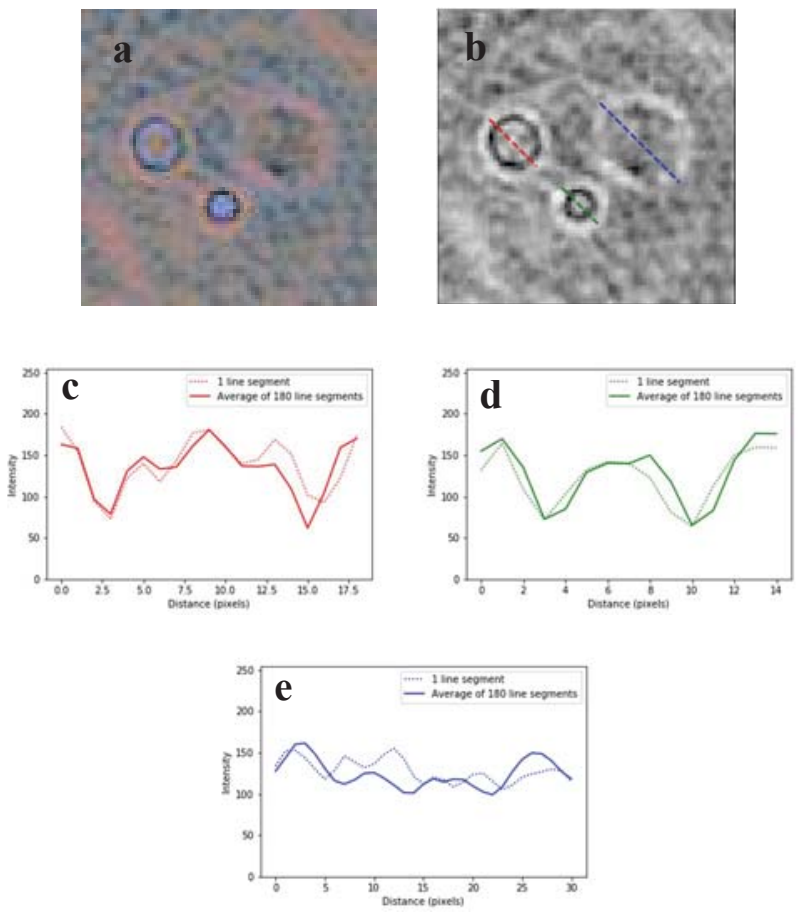

Fig. 3. (a) Colored reconstructed image. (b) Amplitude of the reconstructed images with line segments. (c-e) Intensity profiles through line segments on two live and one dead cells, respectively.
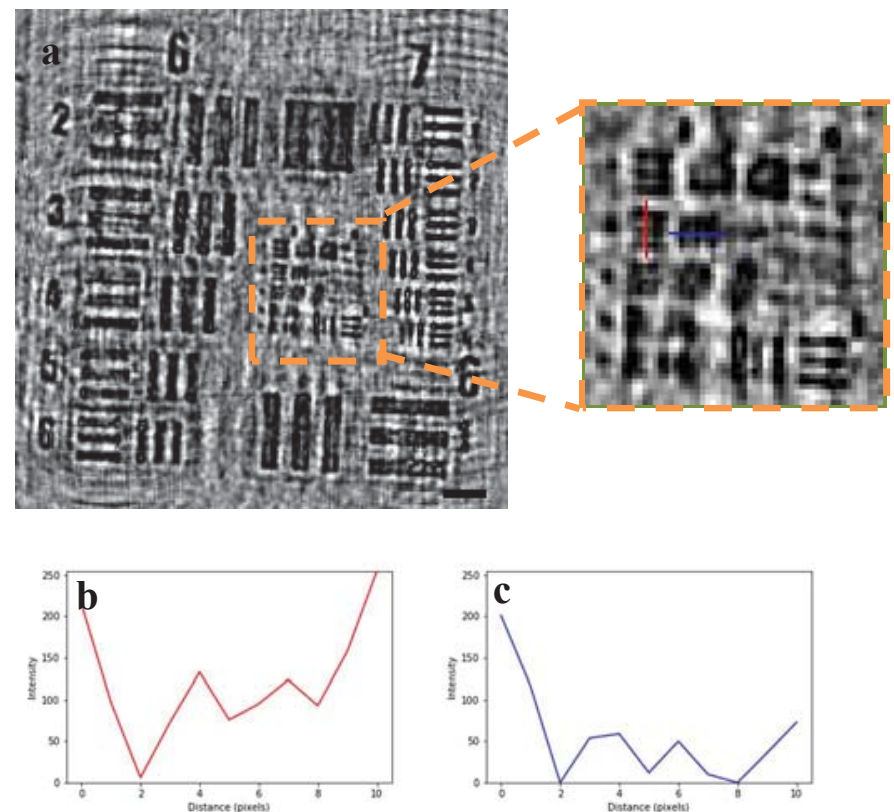

Fig. 4. (a) Reconstructed USAF 1951 test chart image. (b, c) Intensity line profiles line profile on horizontal and vertical bars of G:8-E:3, respectively.

\section{CONCLUSION}

We introduced a low-cost, low-maintenance, easy-to-use and portable microscopy platform that can be used for lensless imaging of cells for space biotechnology applications. This platform can tolerate impacts and heat variations, since sensitive and expensive optical elements are not required. The platform offers high field of view $\left(21.7 \mathrm{~mm}^{2}\right)$ and high resolution $(1.55 \mu \mathrm{m})$ quantitative imaging. Moreover, live/dead cell analysis can be realized on amplitude and phase 
images of reconstructed holograms captured on the platform. The presented platform could allow new opportunities in cellular imaging in space environment with all its advantages and capabilities.

\section{ACKNOWLEDGMENT}

We would like thank to Izmir Institute of Technology (IZTECH) for the financial support (2017IYTE78), the members of Laboratory of Micro and Nanosystems at IZTECH for useful discussions and technical staffs of MultiMedia Lab at IZTECH for operating the laser cutter.

\section{REFERENCES}

[1] E. Ozcivici, "Effects of spaceflight on cells of bone marrow origin," Turk J Haematol, vol. 30, pp. 1-7, Mar 2013.

[2] L. Vico and A. Hargens, "Skeletal changes during and after spaceflight," Nature Reviews Rheumatology, vol. 14, p. 229 , 03/21/online 2018.

[3] N. Foray, M. Maalouf, and M. Durante, "Biological Effects of Space Radiation on Human Cells: History, Advances and Outcomes," Journal of Radiation Research, vol. 52, pp. 126-146, 2011

[4] M. Anil-Inevi, S. Yaman, A. A. Yildiz, G. Mese, O. Yalcin-Ozuysal, H. C. Tekin, et al., "Biofabrication of in situ Self Assembled 3D Cell Cultures in a Weightlessness Environment Generated using Magnetic Levitation," Scientific Reports, vol. 8, p. 7239, 2018/05/08 2018.
[5] E. Ozcivici and S. Judex, "Trabecular bone recovers from mechanical unloading primarily by restoring its mechanical function rather than its morphology," Bone, vol. 67, pp. 122-129, 2014/10/01/ 2014.

[6] E. Ozcivici, W. Zhang, L. R. Donahue, and S. Judex, "Quantitative trait loci that modulate trabecular bone's risk of failure during unloading and reloading," Bone, vol. 64, pp. 25-32, 2014/07/01/ 2014.

[7] Yumpu.com, "Space Transportation Costs Trends in Price per Pound to Orbit," yumpu.com. [Online].

Available: https://www.yumpu.com/en/document/read/36996100/ space-transportation-costs-trends-in-price-per-pound-to-orbit-/4. [Accessed: 11-Apr-2019].

[8] A. C. Sobieranski, F. Inci, H. C. Tekin, M. Yuksekkaya, E. Comunello, D. Cobra, et al., "Portable lensless wide-field microscopy imaging platform based on digital inline holography and multi-frame pixel super-resolution," Light: Science \&Amp; Applications, vol. 4, p. e346, 10/23/online 2015.

[9] K. Delikoyun, E. Ozcivici, H.C. Tekin, "Düşük Maliyetli Ve Portatif Merceksiz Holografik Mikroskobi Platformu İle Hücresel Görüntüleme.” 23. Biyomedikal Bilim ve Teknoloji Sempozyumu, 2018, İstanbul, Turkey.

[10] O. Baskan, G. Mese, and E. Ozcivici, "Low-intensity vibrations normalize adipogenesis-induced morphological and molecular changes of adult mesenchymal stem cells," Proceedings of the Institution of Mechanical Engineers, Part H: Journal of Engineering in Medicine, vol. 231, pp. 160-168, 2017. 